\title{
MULTICOMMODITY CAPACITATED NETWORK DESIGN
}

\section{Bernard Gendron}

Département d'informatique

et de recherche opérationnelle

Centre de recherche sur les transports

Université de Montréal

Teodor Gabriel Crainic

Département des sciences administratives

Université du Québec à Montréal

Centre de recherche sur les transports

Université de Montréal

\section{Antonio Frangioni}

Dipartimento di informatica

Università di Pisa

May 1997 


\begin{abstract}
This paper presents a comprehensive survey of models and algorithms for multicommodity capacitated network design problems, which are mostly encountered in telecommunications and transportation network planning. These problems are important not only due to the major relevance of their applications, but also because they pose considerable modeling and algorithmic challenges. We present a general arc-based model, describe useful alternative formulations and survey the literature on simplex-based cutting plane and Lagrangean relaxation approaches. We then focus on our own contributions that develop and compare several relaxation methods for a particular case of this model, the fixed-charge problem. These methods are based on Lagrangean relaxation and nondifferentiable optimization techniques, namely, the subgradient and bundle approaches. Our experimental results, while very encouraging, indicate that solving efficiently these difficult problems requires a judicious combination of cutting planes, Lagrangean relaxation methods and sophisticated heuristics. In addition, due to their inherent decomposition properties, these techniques can be adapted to parallel computing environments, which is highly desirable in order to solve realistically sized instances.
\end{abstract}

Key words : Multicommodity capacitated network design, cutting planes, Lagrangean relaxation, non-differentiable optimization, parallel computing.

\title{
Résumé
}

Cet article présente une revue de la littérature sur les modèles et les méthodes de résolution de problèmes de conception de réseaux avec capacités. Ces problèmes sont importants non seulement en raison de leurs applications en planification de réseaux de transport et de télécommunications, mais également parce qu'ils posent des défis considérables. Nous présentons un modèle général, ainsi que d'autres formulations alternatives intéressantes, et nous passons en revue les travaux portant sur les méthodes de coupes et de relaxation lagrangienne. Nous décrivons également nos propres contributions, dans lesquelles nous développons et comparons plusieurs méthodes de relaxation pour un cas particulier, le problème avec coûts fixes. Ces méthodes sont basées sur la relaxation lagrangienne et l'optimisation non-différentiable, en particulier les algorithmes de sous-gradients et de faisceaux. Nos résultats expérimentaux, bien qu'encourageants, suggèrent que les méthodes les plus prometteuses consistent à combiner les méthodes de coupes et de relaxation lagrangienne avec des heuristiques sophistiquées, et d'adapter ces méthodes à des environnements parallèles, afin de résoudre efficacement des exemplaires de grande taille.

Mots-clés : Conception de réseaux avec capacités, méthodes de coupes, relaxation lagrangienne, optimisation non-différentiable, calcul parallèle. 


\section{Introduction}

Network design models have wide applications in telecommunications and transportation planning; See, for exemple, the survey articles by Magnanti and Wong [55], Minoux [56], chapter 16 of the book by Ahuja, Magnanti and Orlin [1], section 13 of Ahuja, Magnanti, Orlin and Reddy [2]. In particular, Gavish [30] and Balakrishnan, Magnanti, Shulman and Wong [8] present reviews of important applications in telecommunications. In many of these applications, it is required to send flows (which may be fractional) to satisfy demands given arcs with existing capacities, or to install, in discrete amounts, additional facilities with fixed capacities. In doing so, one pays a price not only for routing flows, but also for using an arc or installing additional facilities. The objective is then to determine the optimal amounts of flows to be routed and the facilities to be installed.

These capacitated network design problems are notoriously difficult, unlike their uncapacitated counterparts for which very efficient specialized algorithms have been devised (see in particular the dual-ascent method of Balakrishnan, Magnanti and Wong [9]). Many reasons explain this phenomenon, among which is the fact that LP relaxations of multicommodity flow formulations generally do not provide tight lower bounds. In addition, the LPs of these formulations are often highly degenerate, which makes their resolution by simplex methods unattractive. Finally, even finding feasible solutions for some of these problems might be a difficult task. To illustrate these points, consider a particular case of the network design problem, the minimum spanning tree problem. As is well-known, the uncapacitated version of the problem is easily solved by greedy algorithms, while the capacitated one is $N P$-hard and very difficult to solve in practice (see Hall [40] and the references therein).

Our objective is twofold: First, to present a survey of models and methods for capacitated network design and second, to describe our contributions on a particular case, the fixed-charge problem. The survey is the object of Section 2, which presents a general arc-based model, discusses interesting alternative formulations, and outline the solution approaches proposed in the literature. Section 3 reviews our work on the fixed- 
charge problem, which compares several relaxations and discusses computational results of bounding procedures (some of the material presented is based on Gendron [32], Gendron and Crainic $[33,36]$ ). In the Conclusion, we summarize this work and discuss future research directions.

\section{Survey}

\subsection{Arc-Based Formulation}

Given a directed graph $G=(N, A)$, a set of commodities $K$ to be routed according to known demands and a set of facilities $L$ to be installed on each arc, the problem consists in routing flows and installing facilities at minimum cost. The objective is therefore to minimize the sum of flow costs and design costs, the latter being charged whenever an arc is used or additional facilities are installed on it. The flow cost per unit of commodity $k$ on $\operatorname{arc}(i, j)$ is denoted $c_{i j}^{k}$, while the design cost for each facility $l$ installed on arc $(i, j)$ is denoted $f_{i j}^{l}$. Both costs are assumed to be nonnegative. To each commodity $k$, we associate origins $O(k)$, destinations $D(k)$ and transshipment nodes $T(k)$. Each origin $i \in O(k)$ supplies $o_{i}^{k}>0$ to the network, while each destination $i \in D(k)$ has a demand $d_{i}^{k}>0$ for commodity $k$. An upper bound $b_{i j}^{k}$ on the amount of flow of commodity $k$ that may pass through arc $(i, j)$ may be imposed. Similarly, there can be an upper bound on the number of facilities $l$ installed on arc $(i, j)$, which we denote $h_{i j}^{l}$. In addition, the total flow on each arc $(i, j)$, which can be weighted for each commodity $k$ by a constant $e_{i j}^{k}$, cannot exceed the capacity on that arc, which consists of an existing capacity $v_{i j}$ and the capacities of the installed facilities, each facility $l$ having a fixed capacity $u_{i j}^{l}$.

To formulate the problem, we introduce continuous flow variables $x_{i j}^{k}$, which reflect routing decisions for each arc $(i, j)$ and each commodity $k$, and integer design variables $y_{i j}^{l}$, which represent the number of facilities $l$ installed on $\operatorname{arc}(i, j)$. The arc-based model is then given by:

$$
\min \sum_{k \in K} \sum_{(i, j) \in A} c_{i j}^{k} x_{i j}^{k}+\sum_{l \in L} \sum_{(i, j) \in A} f_{i j}^{l} y_{i j}^{l}
$$




$$
\begin{gathered}
\sum_{j \in N^{+}(i)} x_{i j}^{k}-\sum_{j \in N^{-}(i)} x_{j i}^{k}=\left\{\begin{array}{cl}
o_{i}^{k} & i \in O(k) \\
-d_{i}^{k} & i \in D(k) \\
0 & i \in T(k)
\end{array} \quad i \in N, k \in K\right. \\
0 \leq x_{i j}^{k} \leq b_{i j}^{k} \quad(i, j) \in A, k \in K \\
\sum_{k \in K} e_{i j}^{k} x_{i j}^{k} \leq v_{i j}+\sum_{l \in L} u_{i j}^{l} y_{i j}^{l} \quad(i, j) \in A \\
A y \leq g \\
0 \leq y_{i j}^{l} \leq h_{i j}^{l} \quad(i, j) \in A, l \in L \\
y_{i j}^{l} \text { integer } \quad(i, j) \in A, l \in L .
\end{gathered}
$$

Each set of constraints is self-explanatory, except for the side constraints (5) associated to the design variables. They can be used to ensure that the design does not exceed a limited budget, or to enforce topological restrictions, such as, for exemple, that the chosen design must be a spanning tree.

Some questions arise from our rather vague description of the problem. First, what constitutes a facility? In telecommunications, facilities may provide, for exemple, high bandwidth point-to-point connections, each transmitting information at different rates (see for example, Magnanti, Mirchandani and Vachani [52], Bienstock and Günlük [18]). In transportation, facilities can represent different types of vehicles used to transport freight (see for example, Kim and Barnhart [47]). Note that we can model the problem without explicitly introducing facilities, by allowing parallel arcs, each of which represents a different facility [2].

A second important issue concerns the nature of the commodities. Either they represent distinct physical goods or, more frequently, they are used to model origin-destination (O-D) pairs. Since the number of O-D pairs can be rather high (in a network with demands between each pair of nodes, there are $O\left(|N|^{2}\right)$ such commodities), some authors prefer, for computational reasons, to represent a commodity as an origin (or a destination). The LP relaxation of this last formulation is usually much easier to solve, but it is also more difficult to identify inequalities that tighten the lower bound (Rardin and Wolsey [60] shed some light on this topic). In general, however, the choice of how to define a commodity should depend on the ability to generate tight valid inequalities, which 
is, in turn, related to the method used to solve the problem. For example, simplex-based approaches might be very sensitive to the size of the LP, and the second modeling option is then perfectly justified $[17,18]$. On the contrary, dual-based methods, such as Lagrangean relaxation and dual-ascent strategies, usually benefit from higher disaggregation.

To qualify the complexity of the problem, it is useful to consider particular cases. As mentioned in the Introduction, efficient procedures exist for uncapacitated problems [9]. For the fixed-charge uncapacitated problem, it is noteworthy that the LP relaxation polytope of the so-called strong formulation is quasi-integral [44], in the sense that every edge of the convex hull of integral points is also an edge of the polytope itself. Such a property is unlikely to hold for the capacitated case. Also, it is much easier for uncapacitated problems to obtain feasible solutions with classical heuristics [55]. Another interesting special case arises when there is only one commodity. In general, the presence of capacities makes the problem difficult. In particular, Magnanti and Mirchandani [50] study the following problem, defined on an undirected graph: There are no flow costs, a single commodity defined as an O-D pair, and two types of facilities, one adding a capacity of 1 per unit, and the other contributing to a capacity of $C>1$ per unit. In addition, there are no side constraints of type (5). They show that if there are existing capacities, or the upper bounds on either the flow variables or the design variables are not redundant (we note that this last case includes the fixed-charge problem), the resulting problem is strongly $N P$-hard, in the sense that the existence of a pseudo-polynomial algorithm for it would imply $P=N P$.

\subsection{Other Formulations}

In addition to the multicommodity directed model, many other formulations have been used, depending on the problem context. We have just seen an example defined on an undirected graph, a situation that happens often in telecommunications. Note however that even if the graph is undirected, flows are generally directed. Also, for some problems defined on undirected graphs, it is possible to derive a directed formulation (for examples, 
see Magnanti and Wolsey [54] for network design problems on trees, and Magnanti and Raghavan [53] for a network design problem with low connectivity constraints).

A number of applications might be modeled by using path-based formulations, in particular those that prescribe the flow between any O-D pair to follow a single path (this is called the nonbifurcated network design problem, as in Gavish and Altinkemer [31], and Barahona [13]). Although path-based formulations have received some attention for multicommodity flow $[14,24]$ and integer multicommodity flow [15], they have been seldom used for network design. Very early, Rardin and Choe [59] compared the arc-based and path-based formulations of the multicommodity capacitated fixed-charge problem. They show that, with respect to their strong LP relaxations, no formulation is better than the other. For the uncapacitated case, however, they show that the arc-based LP relaxation provides a tighter bound than the path-based one. The polyhedral structure of the path-based uncapacitated fixed-charge problem has also been studied by Balakrishnan [4]. More recently, Crainic, Gendreau and Farvolden [21] used the path-based formulation of the capacitated fixed-charge problem in a combined column generation-tabu search heuristic approach, where neighbors are defined by simplex pivots. Their results have provided some of the best known upper bounds for many difficult instances (in Section 3, we use them to qualify lower bounds obtained through relaxations).

Problems without flow costs can also be modeled using cut-based formulations (these problems arise frequently in telecommunications). An important special case is the design of survivable networks, where between each pair of nodes the design should include a prespecified number of edge-disjoint paths (these are the edge survivability requirements; similarly, node survivability constraints can be defined). For these problems, most authors have used cut-based models in conjunction with cutting plane algorithms (see Grötschel, Monma and Stoer [39] and the references therein). An exception is the arc-based formulation proposed by Magnanti and Raghavan [53] for low connectivity problems (the number of edge-disjoint paths between any pair of nodes never exceed two). All these survivable problems are uncapacitated, except the model studied by Stoer and Dahl [61], which also uses a cut-based approach. More recently, researchers have studied 
cut-based models for more general capacitated network design problems $[13,16,57]$. Network design problems on trees are also amenable to so-called packing-based formulations [54].

All these modeling approaches can handle additional requirements that are often encountered in practice. We give a brief list of some of these requirements, with pointers to the literature indicating that they appear in some form or another in the listed papers (note however that some of these problems are not capacitated): nonconvex piecewise linear costs [5, 10]; queueing costs [31]; node location [10]; capacities on the nodes [25]; dynamic multiperiod problems [20]; generalized flows [8]; hierarchical networks [6, 7].

\subsection{Solution Approaches}

To solve capacitated network design problems, researchers have focused on three different approaches: simplex-based cutting plane methods, Lagrangean relaxation and heuristics. Each approach has advantages and drawbacks, which we briefly outline. Simplex-based cutting plane approaches benefit from sophisticated and widely available codes for solving LPs, and they offer an opprtunity for continuous improvement in the lower bounds through the identification of new strong valid inequalities. Moreover, the process of generating these inequalities is now being automated (see for example, Balas, Ceria and Cornuéjols [11, 12]). However, these methods generally do not exploit any particular structure and the LPs can become very large. In addition, for multicommodity capacitated network design, the separation problems for some of the inequalities we outline below are very difficult and the LPs are often highly degenerate [18]. Lagrangean relaxation, on the contrary, exploits the structure of the problem and also facilitates the design of heuristics, by observing the integrality requirements. However, optimizing the Lagrangean dual can be a hard task. For example, traditional subgradient methods [48] sometimes display a zigzagging behavior or even worse, they can stop far from the optimal solution. This phenomenon, due to numerically instable behavior, is called jamming. Another disadvantage of Lagrangean relaxation is that the addition of valid inequalities often destroys the structure of the Lagrangean subproblem. In order to preserve this 
structure, inequalities can be appended to the model by dualizing them (as examples, see Gavish [29], Gavish and Altinkemer [31], Fisher [26], Lucena [49], Gouveia [38]). This approach is very promising but dualization of a large number of constraints can make the Lagrangean dual even more difficult to solve. Finally, heuristics are certainly required to solve hard problems and, when tailored to particular classes of problems, they have shown exceptional practical successes. However, they often lack theoretical justification and, in the absence of tight lower bounding procedures, it is very difficult to assess their performance.

We now briefly review the efforts of the research community in trying to solve capacitated network design problems by cutting planes and Lagrangean relaxation (heuristic methods are described in Crainic, Gendreau and Farvolden [21]). Rardin and Choe [59] and Rardin [58] use Lagrangean relaxation with respect to the constraints linking flow and design variables (which, in the remainder, we call linking constraints). Dual-ascent and subgradient schemes are proposed to optimize the resulting Lagrangean dual, but computational results are shown only for uncapacitated problems. A similar Lagrangean relaxation is proposed by Balakrishnan [3] who also devise a dual-ascent scheme. Again, computational results are shown only for uncapacitated instances. Helgason [43] reports disappointing results obtained by a Lagrangean relaxation/subgradient approach, where flow conservation constraints are dualized. Very recently, this approach has been used by Holmberg and Yuan [45] to solve the multicommodity capacitated fixed-charge problem. Interesting results were obtained by a heuristic that combines subgradient optimization and branch-and-bound.

Whereas all the models described in the previous paragraph are for fixed-charge problems, Magnanti, Mirchandani and Vachani [50,52] are among the first to study a version of the capacitated network design problem with facilities to be installed on the arcs. The problem is defined on an undirected network, but the flow is directed. There are no flow costs, no existing capacities and two types of facilities, one contributing one unit of capacity, the other $C>1$ units of capacity. The capacity constraints limit the total flow of all commodities in both directions. (This problem is the multicommodity version 
of the single-commodity one we have described earlier; see Magnanti and Mirchandani [50].) The authors compare a Lagrangean relaxation with respect to flow conservation constraints with a cutting plane approach. Three types of valid inequalities are used. First, cutset inequalities, which involve only design variables, are based on the following rationale: Across any cut, there should be enough capacity installed to satisfy demands. These basic inequalities are lifted by using modulo arithmetic arguments, and the resulting inequalities are shown to define facets of the convex hull under mild conditions. The second class of inequalities, multicutset (also called partition), are based on the same rationale, except that partitions of the set of nodes into more than two subsets are being considered. The authors also derive a third class of valid inequalities, called arc residual capacity inequalities, that involve both design and flow variables. They combine the linking constraints with the trivial inequalities stating that the sum of flows of any commodity-subset on any arc cannot exceed the total demand for this commodity-subset. By lifting these inequalities using again modulo arithmetic arguments, it can be shown that the resulting inequalities are facet-defining. The authors also prove an important result: Appending these arc residual capacity inequalities to the LP relaxation provides the same lower bound as the Lagrangean relaxation with respect to flow conservation constraints. In their computational results, the authors show that small-size cutset inequalities (with cardinality of one of the subset of nodes not exceeding two) are more effective than arc residual capacity inequalities in reducing the integrality gaps. As a consequence, the lower bound generated by the cutting plane approach is much tighter than the one obtained by Lagrangean relaxation.

Bienstock and Günlük [18] study a similar problem, except that flow costs and existing capacities may be included and, in addition, the total flow in each direction is limited by the capacity. Another difference with the previous model is that a commodity is defined as an origin and not an O-D pair. The authors devised a cutting plane method based on three types of valid inequalities, using the same ideas as above: cutset, multicutset and flow-cutset (this last class involves both flow and design variables). As pointed out by the authors, although the ideas behind the derivation of these inequalities are similar 
to those presented by Magnanti, Mirchandani and Vachani, lifting and facet-proofs can differ significantly. The authors report experiments on two sets of real-life data, both having demands between each pair of nodes. The first set has 15 nodes, 22 edges, large demands, flow costs and existing capacities. The second set has 16 nodes, 49 edges, small demands, no flow costs nor existing capacities. The instances in the first set were easily solved, and because the networks were small and sparse, there was no need to solve the separation problems: Interesting cutsets were simply enumerated. It was a quite different story with the second data set. For one instance in this set, the best lower bound was $20 \%$ away from the optimal value, and before branch-and-bound exhausted all available memory, the gap was still about $10 \%$. The authors advance one explanation for this behavior: Since demands are small, the capacity installed is also very small, resulting in many fractional values. However, the authors could exploit an important problem characteristic: Since there are no existing capacities, facilities must be installed on at least $|N|-1$ edges in order to have a connected network. When appending these spanning tree inequalities, the new lower bound was only $3.4 \%$ from the optimal value and branchand-bound could solve the problem in about 15 minutes. Note however that an instance derived from this one by adding flow costs could be solved only by a parallel branchand-bound code [23] (using as starting LP the formulation with all the cuts appended) in approximately 10 hours (2.4 million nodes were generated).

\section{Relaxations for the Fixed-Charge Problem}

\subsection{Arc-Based Formulation}

Using the same notation as in the previous section, we can derive the arc-based formulation of the multicommodity capacitated fixed-charge problem:

$$
\begin{gathered}
\min \sum_{k \in K} \sum_{(i, j) \in A} c_{i j}^{k} x_{i j}^{k}+\sum_{(i, j) \in E} f_{i j} y_{i j} \\
\sum_{j \in N^{+}(i)} x_{i j}^{k}-\sum_{j \in N^{-}(i)} x_{j i}^{k}=\left\{\begin{array}{cl}
o_{i}^{k} & i \in O(k) \\
-d_{i}^{k} & i \in D(k) \\
0 & i \in T(k)
\end{array} \quad i \in N, k \in K \quad\left(\nu_{i}^{k}\right)\right.
\end{gathered}
$$




$$
\begin{gathered}
0 \leq x_{i j}^{k} \leq b_{i j}^{k} \quad(i, j) \in A, k \in K \\
\sum_{k \in K} x_{i j}^{k} \leq u_{i j} \quad(i, j) \in A \quad\left(\gamma_{i j}\right) \\
\sum_{k \in K} x_{i j}^{k} \leq u_{i j} y_{i j} \quad(i, j) \in E \quad\left(\alpha_{i j}\right) \\
x_{i j}^{k} \leq b_{i j}^{k} y_{i j} \quad(i, j) \in E, k \in K \quad\left(\beta_{i j}^{k}\right) \\
0 \leq y_{i j} \leq 1 \quad(i, j) \in E \\
y_{i j} \text { integer } \quad(i, j) \in E .
\end{gathered}
$$

In this model, $E \subseteq A$ represents the set of design arcs. If some flow is routed through a design arc $(i, j)$, a fixed charge $f_{i j}>0$ is incurred in addition to routing costs. We assume there is an existing capacity $u_{i j}$ on each arc. Note that constraints (12) not only ensure capacity requirements for the design arcs are respected, they also force the flow of any commodity to be 0 if the arc is not chosen in the design. Constraints (13) achieve the same objective; Therefore, they are completely redundant. However, as we will see shortly, these constraints can significantly improve the lower bounds obtained through relaxations. In the remainder, constraints (12) and (13) will be called weak and strong linking constraints, respectively.

\subsection{Relaxations}

We define two continuous relaxations, obtained by dropping integrality requirements, and five Lagrangean relaxations, defined by dualization of sets of constraints. The Lagrangean relaxations are motivated by two objectives: First, to derive a Lagrangean subproblem that is easy to solve (ideally, solvable in polynomial time); second, to minimize the number of dualized constraints. There are two obvious reasons for aiming towards this last objective: We want a Lagrangean subproblem that is as close as possible to the original formulation, and also desire to minimize the number of Lagrangean multipliers.

\section{Weak Relaxation (W)}

This continuous relaxation is derived by dropping the strong linking constraints and the integrality requirements. Since the fixed costs are positive, the weak linking constraints 
then hold at equality in any optimal solution. Therefore, the weak relaxation can be rewritten as:

$$
Z(W)=\min \sum_{k \in K} \sum_{(i, j) \in A / E} c_{i j}^{k} x_{i j}^{k}+\sum_{k \in K} \sum_{(i, j) \in E}\left(c_{i j}^{k}+f_{i j} / u_{i j}\right) x_{i j}^{k}
$$

subject to constraints (9) to (11). This is a multicommodity minimum cost network flow problem (MFP).

\section{Strong Relaxation (S)}

This continuous relaxation is obtained by dropping the integrality constraints. The resulting subproblem is a potentially large-scale LP that can be viewed as an MFP with side variables and side constraints.

\section{Flow Relaxation (F)}

This Lagrangean relaxation is obtained by dualizing constraints (11) to (13). The resulting Lagrangean dual is:

$$
Z(F)=\max _{\gamma, \alpha, \beta \geq 0}\left\{-\sum_{(i, j) \in A} \gamma_{i j} u_{i j}+Z(F X)+Z(F Y)\right\}
$$

where $Z(F X)$ is defined as

$$
Z(F X)=\min \sum_{k \in K} \sum_{(i, j) \in A / E}\left(c_{i j}^{k}+\gamma_{i j}\right) x_{i j}^{k}+\sum_{k \in K} \sum_{(i, j) \in E}\left(c_{i j}^{k}+\gamma_{i j}+\alpha_{i j}+\beta_{i j}^{k}\right) x_{i j}^{k}
$$

subject to constraints $(9)$ to $(10)$, and $Z(F Y)$ is defined as

$$
Z(F Y)=\min _{y \in\{0,1\}^{|E|}} \sum_{(i, j) \in E}\left(f_{i j}-\alpha_{i j} u_{i j}-\sum_{k \in K} \beta_{i j}^{k} b_{i j}^{k}\right) y_{i j}
$$

The Lagrangean subproblem, therefore, decomposes into $|K|$ minimum cost network flow problems and one problem solvable by inspection.

\section{Multicommodity Flow Relaxation (MF)}

This relaxation is derived by dualizing the weak and strong linking constraints but not

the capacity constraints as in the previous relaxation. The resulting Lagrangean dual is:

$$
Z(M F)=\max _{\alpha, \beta \geq 0}\{Z(M F X)+Z(F Y)\}
$$


where $Z(M F X)$ is defined as

$$
Z(M F X)=\min \sum_{k \in K} \sum_{(i, j) \in A / E} c_{i j}^{k} x_{i j}^{k}+\sum_{k \in K} \sum_{(i, j) \in E}\left(c_{i j}^{k}+\alpha_{i j}+\beta_{i j}^{k}\right) x_{i j}^{k}
$$

subject to constraints (9) to (11), and $Z(F Y)$ is defined as in (19). The Lagrangean subproblem separates into an MFP and a problem solvable by inspection.

\section{Knapsack Relaxation (K)}

This relaxation is obtained by dualizing the flow conservation constraints. The resulting Lagrangean dual is:

$$
\begin{gathered}
Z(K)=\max _{\nu}\left\{\sum_{k \in K}\left(\sum_{i \in O(k)} \nu_{i}^{k} o_{i}^{k}-\sum_{i \in D(k)} \nu_{i}^{k} d_{i}^{k}\right)+\right. \\
\left.\min _{(x, y) \in(X, Y)} \sum_{k \in K} \sum_{(i, j) \in A}\left(c_{i j}^{k}+\nu_{j}^{k}-\nu_{i}^{k}\right) x_{i j}^{k}+\sum_{(i, j) \in E} f_{i j} y_{i j}\right\}
\end{gathered}
$$

where the set $(X, Y)$ is defined by constraints (10) to (15). After solving $|A|$ continuous knapsack problems:

$$
\begin{gathered}
g_{i j}=\min \sum_{k \in K}\left(c_{i j}^{k}+\nu_{j}^{k}-\nu_{i}^{k}\right) x_{i j}^{k} \\
\sum_{k \in K} x_{i j}^{k} \leq u_{i j} \\
0 \leq x_{i j}^{k} \leq b_{i j}^{k} \quad k \in K,
\end{gathered}
$$

the Lagrangean subproblem can be solved by inspection as:

$$
\sum_{(i, j) \in A / E} g_{i j}+\min _{y \in\{0,1\}^{|E|}} \sum_{(i, j) \in E}\left(f_{i j}+g_{i j}\right) y_{i j}
$$

\section{Flow-Knapsack Relaxation (FK)}

To define this relaxation, we first introduce new variables $w_{i j}^{k}$, which represent copies of the flow variables, and then decouple flow conservation constraints and linking constraints by reformulating the problem as:

$$
\begin{gathered}
\min \sum_{k \in K} \sum_{(i, j) \in A} c_{i j}^{k} x_{i j}^{k}+\sum_{(i, j) \in E} f_{i j} y_{i j} \\
\sum_{j \in N^{+}(i)} x_{i j}^{k}-\sum_{j \in N^{-}(i)} x_{j i}^{k}=\left\{\begin{array}{cl}
o_{i}^{k} & i \in O(k) \\
-d_{i}^{k} & i \in D(k) \\
0 & i \in T(k)
\end{array} \quad i \in N, k \in K\right.
\end{gathered}
$$




$$
\begin{gathered}
0 \leq x_{i j}^{k} \leq b_{i j}^{k} \quad(i, j) \in A, k \in K \\
\sum_{k \in K} x_{i j}^{k} \leq u_{i j} \quad(i, j) \in A \\
x_{i j}^{k}=w_{i j}^{k} \quad \forall(i, j) \in A, k \in K \quad\left(\sigma_{i j}^{k}\right) \\
0 \leq w_{i j}^{k} \leq b_{i j}^{k} \quad(i, j) \in A, k \in K \\
\sum_{k \in K} w_{i j}^{k} \leq u_{i j} \quad(i, j) \in A \\
\sum_{k \in K} w_{i j}^{k} \leq u_{i j} y_{i j} \quad(i, j) \in E \\
w_{i j}^{k} \leq b_{i j}^{k} y_{i j} \quad(i, j) \in E, k \in K \\
0 \leq y_{i j} \leq 1 \quad(i, j) \in E \\
y_{i j} \text { integer } \quad(i, j) \in A .
\end{gathered}
$$

The Flow-Knapsack Relaxation is then obtained by dropping the capacity constraints (30) and by dualizing constraints (31). The Lagrangean dual can be written as:

$$
Z(F K)=\max _{\sigma}\{Z(F K X)+Z(F K W Y)\}
$$

where $Z(F K X)$ is defined as

$$
Z(F K X)=\min \sum_{k \in K} \sum_{(i, j) \in A}\left(c_{i j}^{k}+\sigma_{i j}^{k}\right) x_{i j}^{k}
$$

subject to constraints (28) and (29), while $Z(F K Y)$ is defined as

$$
Z(F K W Y)=\min -\sum_{k \in K} \sum_{(i, j) \in A} \sigma_{i j}^{k} w_{i j}^{k}+\sum_{(i, j) \in E} f_{i j} y_{i j}
$$

subject to constraints $(32)$ to $(37)$.

\section{Multicommodity Flow-Knapsack Relaxation (MFK)}

We use the same reformulation as above, but here we do not drop constraints (30) and only dualize (31). The resulting Lagrangean dual is:

$$
Z(M F K)=\max _{\sigma}\{Z(M F K X)+Z(F K W Y)\}
$$


where $Z(F K W Y)$ is defined as in (40), while $Z(M F K X)$ is obtained by solving the following MFP:

$$
Z(M F K X)=\min \sum_{k \in K} \sum_{(i, j) \in A}\left(c_{i j}^{k}+\sigma_{i j}^{k}\right) x_{i j}^{k}
$$

subject to constraints $(28)$ to $(30)$.

The lower bounds obtained by these relaxations are compared by the following

\section{Property:}

$$
Z(W) \leq Z(S)=Z(F)=Z(M F)=Z(K)=Z(F K)=Z(M F K)
$$

All relations are easy to prove by noting that the Lagrangean subproblems in the flow, multicommodity flow and knapsack relaxations all have the Integrality property [37] (detailed proofs can also be found in Gendron [32], and Gendron and Crainic [33]).

\subsection{Computational Results}

Computational experiments should first qualify the difference between the weak and the strong lower bounds. If this difference is significant, we then have six different relaxations to compute the most promising bound. Which one is most efficient computationally? Clearly, for Lagrangean relaxations, the answer depends on the method used to optimize the Lagrangean dual. Since their successful application to the traveling salesman problem [41, 42], subgradient algorithms have been widely used in mixed-integer programming. Although they proved to be often useful, sometimes they exhibit zigzagging or, even worse, jamming problems. To handle these difficulties, researchers in the field of nondifferentiable optimization have devised so-called bundle methods [48].

In contrast to subgradient algorithms, bundle methods keep first-order informations in a disaggregated form. These informations, which represent the history of the computations performed so far, are used to update multipliers by computing a tentative direction of ascent. This is performed by solving a semidefinite quadratic programming problem (a very efficient code for solving it is described by Frangioni [27]). Hence, bundle approaches are more difficult to implement than subgradient methods, but they avoid zigzagging by guaranteeing ascent directions. Moreover, for many problems, they have 
shown significant improvements over traditional subgradient methods (some examples are given in Carraresi, Frangioni and Nonato [19]).

Other criteria to consider when assessing the overall efficiency of a given relaxation include, first, its ability to generate feasible solutions by deriving Lagrangean-based heuristic procedures and, second, its decomposition properties which makes it adaptable to a parallel implementation. This last feature is important, since continuous technological improvements in parallel and distributed environments make their exploitation very attractive in the context of solving large-scale and difficult combinatorial problems, such as multicommodity capacitated network design.

In order to partially assess the relaxations, we generated some 234 problems, of various characteristics, using two generators (which are fully described by Gendron and Crainic $[33,36])$. One was used to generate multicommodity bipartite networks, the other general networks with no particular topology. Among this last class, we distinguish single O-D networks, where a commodity is defined as an O-D pair, and multiple O-D networks, for which a commodity may have several origins and destinations. The generators allow to adjust the ratio of fixed costs to routing costs and also the relative importance of capacities.

Our first results [33] support the following conclusions:

- For bipartite and single O-D networks, the improvement in the lower bound due to using the strong relaxation instead of the weak one is very significant (sometimes as high as $40 \%)$.

- The same behavior does not hold for multiple O-D networks, due to the fact that the bound $b_{i j}^{k}$ on the amount of flow of each commodity $k$ that can be routed through every arc $(i, j)$ is very loose. Typically, the only bound known a priori is the sum of all demands for the given commodity. This is in contrast with bipartite and single O-D problems, where tight bounds are known a priori.

- The flow relaxation used in conjunction with a subgradient method provides a very tight approximation to the lower bound of the strong relaxation (on average, less 
than $1 \%$ difference). Moreover, this bound is usually computed in a fraction of the time it took CPLEX 2.1, using the "netopt" option, to compute $Z(S)$.

- For both the knapsack and the flow-knapsack relaxations, the subgradient method exhibited zigzagging and jamming problems. Consequently, the lower bounds given by these two approaches were generally poor.

Using these preliminary results, we then developed bounding procedures based on the flow relaxation [36]. Inspired by the resource-decomposition principle, the upper bounding procedure makes use of the optimal solution $(\bar{x}, \bar{y})$ of the last Lagrangean subproblem and proceeds as follows. First, allocated partial capacities are obtained by solving a projection problem which attempts to satisfy capacity constraints while minimizing the Euclidean distance to $\bar{x}$. The projection decomposes into $|A|$ singlyconstrained quadratic programming problems, for which efficient procedures have been devised [46]. Using the allocated capacities, we then solve a resource-decomposition subproblem, which decomposes into $|K|$ minimum cost network flow problems. The objective of this problem takes into account not only routing costs, but also fixed costs and capacities, in a way similar to the objective of the weak relaxation (16). Given an optimal solution to this problem, an upper bound on the optimal value of the network design problem is obtained. If there is no feasible solution, given the current allocated capacities, the procedure stops. Otherwise, it attempts to improve the current solution by using a generalized add-drop heuristic. First, arcs are sorted according to a criterion that takes into account both the flows and the reduced fixed costs given by the Lagrangean solution. Then, arcs are dropped or added following a dichotomic scheme which, based on the sorting criterion, attempts to identify the most interesting arcs.

Using a set of ten representative instances, Table 1 illustrates the difficulty of standard methods and state-of-the-art software packages to address these problems. All instances belong to the class of single O-D networks, since we are going to show results of the tabu search heuristic of Crainic, Gendreau and Farvolden [21], which is tailored for these problems. The problems are identified by a triplet which indicates the number of nodes, 
arcs and commodities. All these instances are very difficult, since they both have high fixed costs and tight capacity constraints. For each problem, the table displays: $Z(W)$, the lower bound given by the weak relaxation computed by CPLEX 2.1 "netopt"; $Z(S)$, the lower bound given by the strong relaxation computed by CPLEX 2.1 "netopt"; $Z(F)$, the lower bound computed by the flow relaxation using a subgradient method; $Z(R D)$, the upper bound computed by the Lagrangean-based resource-decomposition heuristic; $Z(T S)$, the best upper bound found by the tabu search heuristic; $Z(B B)$, the best upper bound computed by branch-and-bound, based on the strong formulation and using CPLEX 2.1 "optimize" (this is meant to be the optimal value, but for some problems we did not manage to reach the optimum due to memory (64 Megabytes) limitations; an "m" signals these cases). Below each figure, we show, in parentheses, the CPU time, in seconds, on a SPARC Ultra workstation, except for the tabu search heuristic for which CPU time is on a SPARC1000 (which is roughly two times slower than the Ultra). The last column also provides the gap between the best lower and upper bounds. Note that we have not been able to compute $Z(S)$ and $Z(B B)$ for two problems, due to memory limitations (these two figures are replaced by " $\mathrm{M}$ "). Moreover, in one case, indicated by an "X", the resource-decomposition heuristic could not identify any feasible solution.

These results further substantiate the fact that the strong relaxation significantly improves over the weak one. They also show that state-of-the-art LP solvers are not capable of handling problems with a large number of commodities (this is corroborated by the observations of Bienstock and Günlük [17, 18]). In particular, we have observed that the LPs are highly degenerate. In contrast, the flow relaxation is well-adapted to these large-scale problems. However, Lagrangean-based procedures, used alone, are not sufficient to solve these difficult problems. On the one hand, the resource-decomposition heuristic really struggles, especially as the number of commodities increases. On the other hand, while most of the gaps are acceptable, some others are very large.

In view of these results, several questions are of particular interest, most of these we are attempting to address in our actual research:

- Can we find more effective Lagrangean lower bounds by appending valid inequalities 


\begin{tabular}{|c||c|c|c|c|c|c|c|}
\hline problem & $Z(W)$ & $Z(S)$ & $Z(F)$ & $Z(R D)$ & $Z(T S)$ & $Z(B B)$ & gap \% \\
\hline $25,100,10$ & 32262 & 43455 & 43452 & 57688 & 51654 & 49899 & 14.83 \\
& $(1)$ & $(2)$ & $(1)$ & $(2)$ & $(152)$ & $(111)$ & \\
\hline $25,100,30$ & 64037 & 82419 & 82372 & 98618 & 86594 & 85530 & 3.8 \\
& $(1)$ & $(10)$ & $(5)$ & $(4)$ & $(471)$ & $(2541)$ & \\
\hline $100,400,10$ & 33451 & 48375 & 48333 & 115231 & 70063 & 67274 & 39.07 \\
& $(1)$ & $(63)$ & $(6)$ & $(8)$ & $(499)$ & $(6189)$ & \\
\hline $20,230,40$ & 584306 & 633466 & 633432 & 683642 & 644172 & 643036 & 1.51 \\
& $(1)$ & $(5)$ & $(10)$ & $(7)$ & $(1121)$ & $(7181)$ & \\
\hline $20,300,40$ & 546666 & 596839 & 596819 & 704528 & 605398 & 604198 & 1.23 \\
& $(1)$ & $(8)$ & $(12)$ & $(15)$ & $(853)$ & $(312)$ & \\
\hline $20,300,200$ & 84733 & 103633 & 103440 & 332193 & 130715 & $111565 \mathrm{~m}$ & 7.65 \\
& $(5)$ & $(3925)$ & $(67)$ & $(40)$ & $(8110)$ & $(100220)$ & \\
\hline $30,520,100$ & 76710 & 94011 & 93691 & 209087 & 107894 & $98500 \mathrm{~m}$ & 4.77 \\
& $(4)$ & $(2553)$ & $(57)$ & $(59)$ & $(17599)$ & $(56998)$ & \\
\hline $30,520,400$ & 126357 & $\mathrm{M}$ & 149438 & $\mathrm{X}$ & 173344 & $\mathrm{M}$ & 16.00 \\
& $(111)$ & & $(232)$ & $(122)$ & $(61615)$ & & \\
\hline $30,700,100$ & 44811 & 53661 & 53537 & 100850 & 58704 & $56565 \mathrm{~m}$ & 5.41 \\
& $(4)$ & $(1157)$ & $(72)$ & $(95)$ & $(11537)$ & $(26575)$ & \\
\hline $30,700,400$ & 109364 & $\mathrm{M}$ & 127314 & 344615 & 150948 & $\mathrm{M}$ & 18.56 \\
& $(120)$ & & $(301)$ & $(190)$ & $(88310)$ & & \\
\hline
\end{tabular}

Table 1: Lower Bounds, Heuristics and Optimal Values 
to the strong formulation? We have examined this approach in an earlier contribution [33] by adding a limited set of cutset inequalities in such a way that the Lagrangean subproblem decomposes into 0-1 knapsack problems, therefore keeping the subproblems tractable. The improvement, however, was very limited, if not inexistent. As mentioned above, a more promising approach dualizes valid lifted inequalities. The corresponding bound is then at least as good as the strengthened LP and the Lagrangean subproblem's structure is preserved.

- Can we devise more efficient Lagrangean procedures by using bundle methods? We have preliminary computational results showing that bundle approaches are slower than subgradient methods for the flow relaxation. This behavior might be explained by the large number of multipliers generated by this relaxation, which imposes a heavy computational burden on the quadratic problem. We have other preliminary results, soon to be reported, showing that the knapsack relaxation/bundle approach can outperform subgradient methods. Also of great interest is the contribution that bundle methods could bring to the design of more effective Lagrangean heuristics. Along this line, we note that multicommodity flow problems, especially those with a large number of commodities, are now routinely solved at optimality by a specialized bundle algorithm (see Frangioni and Gallo [28], which reports remarkable results for the same test problems). This observation motivates the use of multicommodity flow relaxations to solve our problem. These relaxations have the notable advantage of generating feasible solutions every time a Lagrangean subproblem is solved.

- Can we combine Lagrangean and heuristic approaches to improve the performance of both? Lagrangean heuristics can be used to provide starting solutions to more sophisticated heuristics such as tabu search. Also, lower bounds and dual information can guide the search for promising solutions in a way reminiscent of the branching rules used in branch-and-bound algorithms.

- Can we make effective use of parallel computing to help solve these difficult problems? There are mainly three ways of exploiting parallel computing. The first 
approach accelerates bound computations, notably by exploiting decomposition by commodities (preliminary results in this direction were given by Gendron and Crainic [35]). A second approach would attempt to improve the search for promising solutions by exploring multiple paths in parallel. These approaches can be adapted to either branch-and-bound algorithms [34] or tabu search procedures [22]. Finally, parallel computing might facilitate the task of combining Lagrangean and heuristic methods since, in a distributed environment, one can easily merge two or more programs without forcing them to share common data structures.

\section{Conclusion}

This survey has shown that capacitated network design problems pose considerable modeling and algorithmic challenges. In particular, several formulations (arc-based, pathbased, cut-based) are possible for the same problem and it is not entirely clear which is most attractive. An appropriate answer to this question should take into account the quality of the lower bounds that can be generated through relaxations, as well as the methods used to solve the models. These methods can be divided into three categories: simplex-based cutting plane algorithms, Lagrangean relaxation and heuristics. We have seen, through examples from the literature and from our own research on the fixed-charge problem, that each of these approaches, used alone, is probably insufficient to solve difficult and large-scale instances. Following the presentation of our computational results on the fixed-charge problem, we have described several ways to combine them. In addition, we have identified some interesting research questions and discussed how parallel computing can help to solve these difficult problems.

\section{Acknowledgments}

Financial support for this project was provided by N.S.E.R.C. (Canada) and the Fonds F.C.A.R. (Québec). 


\section{References}

[1] Ahuja R.K., Magnanti T.L. and Orlin J.B. (1993), Network Flows: Theory, Algorithms, and Applications, Prentice-Hall.

[2] Ahuja R.K., Magnanti T.L., Orlin J.B. and Reddy M.R. (1995) Applications of Network Optimization, chapter 1 of Network Models, Handbooks of Operations Research and Management Science, volume 7, 1-83.

[3] Balakrishnan A. (1984), Valid Inequalities and Algorithms for the Network Design Problem with an Application to LTL Consolidation Problem, Ph.D. thesis, Sloan School of Management, Massachusetts Institute of Technology.

[4] Balakrishnan A. (1987), LP Extreme Points and Cuts for the Fixed-Charge Network Design Problem, Mathematical Programming, 39, 263-284.

[5] Balakrishnan A. and Graves S.C. (1989), A Composite Algorithm for a Concave-Cost Network flow Problem, Networks, 19, 175-202.

[6] Balakrishnan A., Magnanti T.L. and Mirchandani P. (1994), A Dual-Based Algorithm for Multi-Level Network Design Problem, Management Science, 40(5), 567-581.

[7] Balakrishnan A., Magnanti T.L. and Mirchandani P. (1994), Modeling and WorstCase Performance Analysis of the Two-Level Network Design Problem, Management Science, 40(7), 846-867.

[8] Balakrishnan A., Magnanti T.L., Shulman A. and Wong R.T. (1991), Models for Planning Capacity Expansion in Local Access Telecommunication Networks, Annals of Operartions Research, 33, 239-284.

[9] Balakrishnan A., Magnanti T.L. and Wong R.T. (1989), A Dual-Ascent Procedure for Large-Scale Uncapacitated Network Design, Operations Research, 37, 716-740. 
[10] Balakrishnan A., Magnanti T.L. and Wong R.T. (1995), A Decomposition Algorithm for Local Access Telecommunications Network Expansion Planning, Operations Research, 43(1), 58-76.

[11] Balas E., Ceria S. and Cornuéjols G. (1993), A Lift-and-Project Cutting Plane Algorithm for Mixed 0-1 Programs, Mathematical Programming 58, 295-324.

[12] Balas E., Ceria S. and Cornuéjols G. (1995), Mixed 0-1 Programming by Lift-andProject in a Branch-and-Cut Framework, working paper, Graduate School of Business, Columbia University.

[13] Barahona F. (1996), Network Design Using Cut Inequalities, SIAM Journal on Optimization 6(3), 823-837.

[14] Barnhart C., Hane C.A., Johnson E.L. and Sigismondi G. (1995), A Column Generation and Partitioning Approach for Multicommodity Flow Problems, Telecommunications Systems, 3, 239-258.

[15] Barnhart C., Hane C.A. and Vance P.H. (1996), Integer Multicommodity Flow Problems, working paper, Center for Transportation Studies, Massachusetts Institute of Technology.

[16] Bienstock D., Chopra S., Günlük O. and Tsai C.-Y. (1995), Minimum-Cost Capacity Installation for Multicommodity Network Flows, working paper, Department of Industrial Engineering and Operations Research, Columbia University.

[17] Bienstock D. and Günlük O. (1995), Computational Experience with a Difficult Mixed-Integer Multicommodity Flow Problem, Mathematical Programming, 68(2), 213-237.

[18] Bienstock D. and Günlük O. (1996), Capacitated Network Design-Polyhedral Structure and Computation, INFORMS Journal on Computing, 8(3), 243-259. 
[19] Carraresi P., Frangioni A. and Nonato M. (1996), Applying Bundle Methods to the Optimization of Polyhedral Functions: An Applications-Oriented Development, working paper, Dipartimento di informatica, Università di Pisa.

[20] Chang S.-G. and Gavish B. (1995), Lower Bounding Procedures for Multiperiod Telecommunications Network Expansion Problems, Operations Research, 43(1), 4357.

[21] Crainic T.G., Gendreau M. and Farvolden J. (1996), Simplex-Based Tabu Search for the Multicommodity Capacitated Fixed Charge Network Design Problem, publication CRT-96-07, Centre de recherche sur les transports, Université de Montréal.

[22] Crainic T.G., Toulouse M. and Gendreau M. (1993), Towards a Taxonomy of Parallel Tabu Search Algorithms, to appear in INFORMS Journal on Computing.

[23] Eckstein J. (1995), Parallel Branch-and-Bound Algorithms for General MixedInteger Programming on the CM-5, SIAM Journal on Optimization, 4(4), 794-814.

[24] Farvolden J.M., Powell W.B. and Lustig I.J. (1993), A Primal Partitioning Solution for the Arc-Chain Formulation of a Multicommodity Network Flow Problem, Operations Research, 41(4), 669-693.

[25] Fetterolf P.C. and Anandalingam G. (1992), A Lagrangean Relaxation Technique for Optimizing Interconnection of Local Area Networks, Operations Research, 40(4), 678-688.

[26] Fisher M.L. (1994), Optimal Solution of Vehicle Routing Problems Using Minimum K-Trees, Operations Research, 42, 393-410.

[27] Frangioni A. (1995), Solving Semidefinite Quadratic Problems within Nonsmooth Optimization Algorithms, working paper, Dipartimento di informatica, Università di Pisa. 
[28] Frangioni A. and Gallo G. (1996), A Bundle Type Dual-Ascent Approach to Linear Multicommodity Min Cost Flow Problems, working paper, Dipartimento di informatica, Università di Pisa.

[29] Gavish B. (1985), Augmented Lagrangean Based Algorithms for Centralized Netowrk Design, IEEE Transactions on Communications 33(12), 1247-1257.

[30] Gavish B. (1991), Topological Design of Telecommunications Networks-Local Access Design Methods, Annals of Operations Research, 33, 17-71.

[31] Gavish B. and Altinkemer K. (1990), Backbone Network Design Tools with Economic Tradeoffs, ORSA Journal on Computing, 2(3), 236-252.

[32] Gendron B. (1994), Nouvelles méthodes de résolution de problèmes de conception de réseaux et leur implantation en environnement parallèle, Ph.D. thesis, Département d'informatique et de recherche opérationnelle, Université de Montréal, publication CRT-94-50, Centre de recherche sur les transports, Université de Montréal.

[33] Gendron B. and Crainic T.G. (1994), Relaxations for Multicommodity Capacitated Network Design Problems, publication CRT-965, Centre de recherche sur les transports, Université de Montréal.

[34] Gendron B. and Crainic T.G. (1994), Parallel Branch-and-Bound Algorithms: Survey and Synthesis, Operations Research, 42(6), 1042-1066.

[35] Gendron B. and Crainic T.G. (1994), Parallel Implementations of Bounding Procedures for Multicommodity Capacitated Network Design Problems, publication CRT94-45, Centre de recherche sur les transports, Université de Montréal.

[36] Gendron B. and Crainic T.G. (1996), Bounding Procedures for Multicommodity Capacitated Fixed Charge Network Design Problems, publication CRT-96-06, Centre de recherche sur les transports, Université de Montréal.

[37] Geoffrion A.M. (1974), Lagrangean Relaxation for Integer Programming, Mathematical Programming Study, 2, 82-114. 
[38] Gouveia L. (1995), A 2n Constraint Formulation for the Capacitated Minimal Spanning Tree Problem, Operations Research, 43(1), 130-141.

[39] Grötschel M., Monma C.L. and Stoer M. (1995), Design of Survivable Networks, chapter 10 of Network Models, Handbooks in Operations Research and Management Science, volume 7, 617-672.

[40] Hall L. (1996), Experience with a Cutting Plane Algorithm for the Capacitated Spanning Tree Problem, INFORMS Journal on Computing, 8(3), 219-234.

[41] Held M. and Karp R.M. (1970), The Traveling Salesman Problem and Minimum Spanning Trees, Operations Research, 18, 1138-1162.

[42] Held M. and Karp R.M. (1971), The Traveling Salesman Problem and Minimum Spanning Trees: Part II, Mathematical Programming, 1, 6-25.

[43] Helgason R.V. (1980), A Lagrangean Relaxation Approach to the Generalized Fixed Charge Multicommodity Minimal Cost Network Flow Problem, Ph.D. thesis, School of engineering and Applied Science, Southern Methodist University.

[44] Hellstrand J., Larsson T. and Migdalas A. (1992), A Characterization of the Uncapacitated Network Design Polytope, Operations Research Letters, 12, 159-163.

[45] Holmberg K. and Yuan D. (1996), A Lagrangean Heuristic Based Branch-and-Bound Approach for the Capacitated Netowrk Design Problem, research report LiTH-MATR-1996-23, Department of Mathematics, Linkoping Institute of Technology.

[46] Kennington J.L. and Helgason R.V. (1980), Algorithms for Network Programming, Wiley.

[47] Kim D. and Barnhart C. (1996), Multimodal Express Shipment Service Design: Models and Algorithms, working paper, Center for Transportation Studies, Massachusetts Institute of Technology. 
[48] Lemaréchal C. (1989), Nondifferentiable Optimization, chapter 7, Optimization, Handbooks in Operations Research and Management Science, volume 1, 529-572.

[49] Lucena A. (1993), Steiner Problem in Graphs: Lagrangean Relaxation and CuttingPlanes, presented at NETFLOW93, San Miniato, Italy, October 3-7 (technical report TR-21/93, Dipartimento di informatica, Università di Pisa, 147-154).

[50] Magnanti T.L. and Mirchandani P. (1993), Shortest Paths, Single Origin-Destination Network Design, and Associated Polyhedra, Networks, 23(2), 103-121.

[51] Magnanti T.L., Mirchandani P. and Vachani R. (1993), The Convex Hull of Two Core Capacitated Network Design Problems, Mathematical Programming, 60, 233250.

[52] Magnanti T.L., Mirchandani P. and Vachani R. (1995), Modeling and Solving the Two-Facility Capacitated Network Loading Problem, Operations Research, 43(1), $142-157$.

[53] Magnanti T.L. and Raghavan S. (1994), A Flow-Based Approach to Low Connectivity Network Design, working paper, Operations Research Center, Massachusetts Institute of Technology.

[54] Magnanti T.L. and Wolsey L.A. (1995), Optimal Trees, chapter 9, Network Models, Handbooks in Operations Research and Management Science, volume 7, 503-615.

[55] Magnanti T.L. and Wong R.T. (1984), Network Design and Transportation Planning: Models and Algorithms, Transportation Science, 18(1), 1-55.

[56] Minoux M. (1989), Network Synthesis and Optimum Network Design Problems: Models, Solution Methods and Applications, Networks, 19, 313-360.

[57] Mirchandani P. (1992), Projections of the Capacitated Network Loading Problem, working paper, Katz Graduate School of Business, University of Pittsburgh. 
[58] Rardin R.L. (1982), Tight Relaxations of Fixed Charge Network Flow Problems, working paper, School of Industrial and Systems Engineering, Georgia Institute of Technology.

[59] Rardin R.L. and Choe U. (1979), Tighter Relaxations of Fixed Charge Network flow Problems, working paper, School of Industrial and Systems Engineering, Georgia Institute of Technology.

[60] Rardin R.L. and Wolsey L.A. (1993), Valid Inequalities and Projecting the Multicommodity Extended Formulation for Uncapacitated Fixed Charge Network Flow Problems, European Journal of Operational Research, 71, 95-109.

[61] Stoer M. and Dahl G. (1994), A Polyhedral Approach to Multicommodity Survivable Network Design, Numerische Mathematik, 68, 149-167. 